\section{Substantia Nigra}

George Leichnetz

Virginia Commonwealth University, Richmond, VA, USA

\section{Definition}

The substantia nigra (SN) is a nucleus in the ventral part of the midbrain tegmentum, which is darkly pigmented (hence its name) because neuromelanin is a by-product of the synthesis of catecholamines, like dopamine. The substantia nigra is the principal source of dopamine in the brain. The SN contains two subdivisions: the pars compacta ( $\mathrm{SNc}$ ), which contains the cell bodies of dopaminergic neurons, and the pars reticulata ( $\mathrm{SNr}$, which contains the cell bodies of GABA- ergic neurons. The SNc projects primarily to the striatum (caudate and putamen), whereas the $\mathrm{SNr}$ projects primarily to the superior colliculus, motor thalamus (VA/VL), and intralaminar nuclei of the thalamus. In Parkinson's disease, there is a substantial loss of dopaminergic neurons in the $\mathrm{SNc}$, which results in a resting tremor, bradykinesia, freezing, and festinating gait.

\section{Cross-References}
Dopamine
- Midbrain
- Parkinson's Disease
- Superior Colliculus
Thalamus

The author is retired. 